\title{
Qual será a oferta de serviços de saúde bucal e estimativa de repasses para a atenção básica nos próximos 30 anos? Um estudo de prospecção
}

\section{What will be the supply of oral health services and estimated transfers to primary care over the next 30 years? A prospective study}

\author{
José Eudes de Lorena Sobrinho ${ }^{1}$ \\ Thaís Regis Aranha Rossi ${ }^{2}$ \\ Petrônio José de Lima Martelli ${ }^{3}$ \\ Sônia Cristina Lima ${ }^{4}$
}

RESUMO: O objetivo deste artigo foi estimar a oferta de serviços de saúde bucal na atenção básica e seus custos em prospecção no período 2020-2050. Trata-se de estudo quantitativo e prospectivo. Para se realizar as prospecções, foram consultadas as seguintes informações: a) Projeção da população brasileira para o período 2020-2060 segundo o IBGE; b) Cobertura populacional da saúde bucal na atenção básica na série histórica 2004-2018 segundo o Ministério da Saúde; c) Os valores repassados pelo governo federal aos municípios para o custeio das equipes de saúde bucal; d) Índice Nacional de Preços ao Consumidor Amplomedido pelo IBGE. Foram previstos três cenários para análise do presente estudo: cenário favorável possível, cenário realista provável e cenário pessimista plausível. No primeiro cenário, foi previsto o aumento no repasse de recursos, respeitando-se a vigência da Emenda Constitucional 95/2016. A estimativa para a quantidade de eSB seria crescente nos próximos 30 anos. No cenário realista provável, considerou-se a manutenção do atual percentual de cobertura pelas equipes de saúde bucal na atenção básica com base em 2018 ao longo dos próximos 30 anos. Os valores de repasse do governo acompanhariam para o futuro a tendência da série histórica. No cenário pessimista plausível vislumbra-se a diminuição oferta e cobertura de serviços de saúde bucal na APS, a partir das recentes mudanças na Política Nacional de Atenção Básica (PNAB). As ameaças às conquistas históricas relativas à construção das respostas do Estado aos problemas de saúde bucal são reais e alarmantes.

\footnotetext{
1 Doutor em Saúde Pública. Professor da Universidade de Pernambuco (UPE) e do Centro Universitário Tabosa de Almeida (Asces-Unita). Docente Permanente do Programa de Pós-Graduação em Hebiatria.

2 Doutora em Saúde Pública. Professora da Universidade do Estado da Bahia (UNEB). Docente permanente do Metrado Profissional em Saúde Coletiva/UNEB.

3 Doutor em Saúde Pública. Professor Adjunto da Universidade Federal de Pernambuco (UFPE).

4 Doutora em Saúde Pública. Professora da Universidade Federal da Bahia. Docente do quadro permanente do

Programa de Pós-graduação do Instituto de Saúde Coletiva/UFBA.
} 
Descritores: Políticas de Saúde, Saúde Bucal, Financiamento governamental

ABSTRACT: The objective of this paper was to estimate the supply of oral health services in primary care and the ir prospecting costs in the period 2020-2050. This is a quantitative and prospective study. To carry out the prospecting, the follow ingin formation was consulted: a) Projection of the Brazilian population for the period 2020-2060 according to the IBGE; b) Population coverage of oral health in primary care in the historical series 2004-2018 according to the Ministry of Health; c) The amounts passed on by the federal governmentto the municipalities to fund oral health teams; d) Broad National ConsumerPrice Index measured by IBGE. Threes cenarios wereforeseen for analysis of this study: possible favorable scenario, probable realistic scenario and plausible pessimistic scenario. In the first scenario, an increase in the transfer off undswasforeseen, respecting the validity of ConstitutionalAmendment 95/2016. The estimate for the amount of eSB would be increasing over thenext 30 years. In the probable realistic scenario, we considered main taining the current percentage of cover age by oral health teams in primary carebasedon 2018 over thenext 30 years. Government transfer values would follow the trend of thehistorical series for the future. In the plausible pessimistics cenario, wecansee a decrease in thesupplyandcoverageof oral health services in PHC, basedon recent changes in the National Policy for Primary Care (PNAB). Threats to historical achievements in building state responses to oral health problems are real and alarming.

Key-words: Health Policy, Oral health, Financing, Government

RESUMEN: El objetivo de este documento fue estimar la oferta de servicios de salud bucal enatención primaria y sus costos de prospecciónenel período 2020-2050. Este es unestudiocuantitativo y prospectivo. Para llevar a cabo laprospección, se consultólasiguienteinformación: a) Proyección de lapoblaciónbrasileña para el período 2020-2060 segúnel IBGE; b) Cobertura poblacional de salud bucal enatención primaria enla serie histórica 2004-2018 segúnelMinisterio de Salud; c) Los montospasados por elgobierno federal a losmunicipios para financiar equipos de salud bucal; d) Índice nacional amplio de preciosal consumidormedido porIBGE. Se previerontresescenarios para elanálisis de este estudio: posibleescenariofavorable, probableescenario realista y escenariopesimistaplausible. Enel primer escenario, se preveíaun aumento enlatransferencia de fondos, respetandola validez de laEnmienda Constitucional 95/2016. La estimación de lacantidad de eSBaumentaríaenlos próximos 30 años. Enelescenario realista probable, consideramos mantenerelporcentajeactual de cobertura por parte de losequipos de salud bucal enatención primaria con base en 2018 durante los próximos 30 años. Los valores de transferenciadelgobiernoseguiríanlatendencia de las series históricas para el futuro. Enelescenariopesimistaplausible, podemos ver ladisminuciónenla oferta y la cobertura de losservicios de salud bucal enla APS, enfunción de loscambiosrecientesenla Política Nacional de Atención Primaria (PNAB). Lasamenazas a los logros históricos enlaconstrucción de respuestasestatales a los problemas de salud oral sonreales y alarmantes.

Palabras-clave: Política de Salud, Salud bucal, Financiación Gubernamental 


\section{INTRODUÇÃO}

Ao longo dos últimos quinze anos, desde a implantação da política nacional de saúde bucal (PNSB) Brasil Sorridente em 2004루, observou-se crescimento da oferta de serviços na atenção básica e especializada, medida através do quantitativo de Equipes de Saúde Bucal (eSB) e Centros de Especialidades Odontológicas (CEO) ${ }^{2}$.

Dados do Ministério da Saúde indicam que eram 8.951 eSB em funcionamento no país em 2004, perfazendo 26,63\% de cobertura populacional. Em 2018 este total foi de 26.712, ou seja, 40,29\% de população coberta ${ }^{2}$. Por consequência, constatou-se melhoria dos indicadores epidemiológicos de prevalência da cárie dentária e edentulismo ${ }^{3}$, embora estes persistam como problemas de grande magnitude no país, com clusters em localidades de maior pobreza e forte influência dos determinantes sociais ${ }^{4}$.

Tendo encontrado um cenário favorável para sua implementação entre 2004 e 2015, a PNSB vem perdendo institucionalização, revelando sua fragilidade e volatilidade às transformações políticas ocorridas no país, emergindo a necessidade de que se torne uma política de Estado ${ }^{5}$.

Reduções no volume de recursos financeiros para a saúde bucal bem como as mudanças no formato de repasses intergovernamentais, provocadas pelos ajustes econômicos realizados pelo governo federal após 2016, vem provocando diminuição na ampliação da oferta de serviços no setor ${ }^{6}$.

Há, portanto,entraves quanto a necessidade de crescimento da oferta de serviços na atenção básica capaz de ampliar o acesso da população, em razão do caráter universal do sistema de saúde brasileiro, e a austeridade fiscal que impõe limites de gastos e congelamento de recursos para os próximos anos ${ }^{7}$.

Estudos de prospecção podem trazer para o foco, problematizar e formular o suposto, o possível e o esperado ${ }^{8}$. A prospecção tem sido empregada de forma sistemática pela indústria farmacêutica, de equipamentos e materiais para cuidados da saúde, entretanto não tão frequente em outras áreas da saúde. Trabalho realizado em 2016, realizou o esforço de esboçar estrategicamente diretrizes para o Sistema de Saúde em 2030.

O objetivo deste artigo é estimar a oferta de serviços de saúde bucal na atenção básica e seus custos em prospecção no período 2020-2050.

\section{METODOLOGIA}

Trata-se de estudo quantitativo e prospectivo que buscou estimar a quantidade de equipes de saúde bucal na atenção básica a serem implantadas no Brasil e seus custos estimados de acordo com a projeção do crescimento da população brasileira no período de 2020 a 2050. 
Para se realizar as prospecções, foram consultadas as seguintes informações: a) Projeção da população brasileira para o período 2020-2060 segundo o Instituto Brasileiro de Geografia e Estatística; b)Cobertura populacional da saúde bucal na atenção básica na série histórica 20042018 segundo a Sala de Apoio à Gestão Estratégica e Participativa do Ministério da Saúde (Quadro 1); c) Os valores repassados pelo governo federal aos municípios para o custeio das equipes de saúde bucal de acordo com as portarias ministeriais ao longo do período 2000-2019 (Quadro 2); d) Índice Nacional de Preços ao Consumidor Amplo (IPCA) medido pelo Instituto Brasileiro de Geografia e Estatística (IBGE) no período 1999-2018.

A partir dos dados do Quadro 1 foi possível calcular a prospecção de Equipes de Saúde Bucal (ESB) no Brasil em 2010 e estimativas para 2020, 2030, 2040 e 2050. Os dados foram coletados a partir do site o Departamento de Atenção Básica do Ministério da Saúde: https://egestorab.saude. gov.br/paginas/acessoPublico/relatorios/relHistoricoCoberturaAB.xhtml

Quadro 1. Número de equipes de saúde bucal e percentual de população coberta no Brasil 2004-2018.

\begin{tabular}{|c|c|c|c|}
\hline Ano & $\begin{array}{c}\text { Número de equipes } \\
\text { de saúde bucal }\end{array}$ & $\begin{array}{c}\text { Percentual de População } \\
\text { coberta }\end{array}$ & População Coberta \\
\hline 2004 & 8.951 & 26,63 & 47.099 .677 \\
\hline 2005 & 12.602 & 34,71 & 62.167 .279 \\
\hline 2006 & 15.086 & 39,85 & 73.399 .925 \\
\hline 2007 & 15.694 & 40,87 & 76.335 .022 \\
\hline 2008 & 17.801 & 39,2 & 74.226 .594 \\
\hline 2009 & 18.982 & 47,5 & 90.073 .201 \\
\hline 2010 & 20.424 & 34,01 & 65.127 .310 \\
\hline 2011 & 21.425 & 35,71 & 68.123 .083 \\
\hline 2012 & 22.203 & 36,73 & 70.655 .082 \\
\hline 2013 & 23.150 & 37,9 & 73.524 .665 \\
\hline 2014 & 24.279 & 37,95 & 76.302 .598 \\
\hline 2015 & 24.467 & 37,82 & 76.704 .752 \\
\hline 2016 & 24.383 & 37,4 & 86.480 .852 \\
\hline 2017 & 25.890 & 39,07 & 83.666 .863 \\
\hline 2018 & 26.712 & 40,29 & \\
\hline
\end{tabular}

Fonte: Ministério da Saúde (BRASIL, 2019).

A prospecção sobre a estimativa de custos das equipes de saúde bucal no Brasil em 2020, 2030, 2040 e 2050 foi realizada, a partir da série histórica de repasses de recursos, estabelecida a partir das portarias que regulamentam o repasse de recursos para a Saúde Bucal, de 2000 a 2019. 
Quadro 2.Portarias e valores estabelecidos para repasse de recursos para financiamento da saúde bucal na atenção básica no período de 2000 a 2019.

\begin{tabular}{|c|c|c|}
\hline Portarias & Modalidade I & Modalidade II \\
\hline $\begin{array}{l}\text { Portaria } \mathrm{n}^{\circ} 1.444 \text { de } 28 \text { de dezembro de } \\
2000\end{array}$ & $\begin{array}{l}\text { Implantação: R } \$ \text { 5.000,00 } \\
\text { Custeio/ano: } \mathrm{R} \$ 13.000,00\end{array}$ & $\begin{array}{l}\text { Implantação: R\$ 5.000,00 } \\
\text { Custeio/ano: R\$ 16.000,00 }\end{array}$ \\
\hline Portaria $^{\circ} 673$ de 03 de junho de 2003 & $\begin{array}{l}\text { Implantação: R\$ } 5.000,00 \\
\text { Custeio/ano: R\$ } 15.600,00\end{array}$ & $\begin{array}{l}\text { Implantação: R\$ 5.000,00 } \\
\text { Custeio/ano: R\$ 19.200,00 }\end{array}$ \\
\hline Portaria $\mathrm{n}^{\circ} 74$ de 20 de janeiro de 2004 & $\begin{array}{l}\text { Implantação: R\$ } 6.000,00 \\
\text { Custeio/ano: R\$ 20.400,00 }\end{array}$ & $\begin{array}{l}\text { Implantação: R } \$ 6.000,00 \\
\text { Custeio/ano: R } 26.400,00\end{array}$ \\
\hline Portaria $\mathrm{n}^{\circ} 650$ de 28 de março de 2006 & $\begin{array}{l}\text { Implantação: R\$ 7.000,00 } \\
\text { Custeio/ano: R \$ 20.400,00 }\end{array}$ & $\begin{array}{l}\text { Implantação: R\$ 7.000,00 } \\
\text { Custeio/ano: R } \$ 26.400,00\end{array}$ \\
\hline Portaria $\mathrm{n}^{\circ} 2.489$ de 21 outubro de 2008 & $\begin{array}{l}\text { Implantação: R\$ 7.000,00 } \\
\text { Custeio/ano: R\$ 22.800,00 }\end{array}$ & $\begin{array}{l}\text { Implantação: R\$ 7.000,00 } \\
\text { Custeio/ano: R \$ 29.400,00 }\end{array}$ \\
\hline $\begin{array}{l}\text { Portaria } \mathrm{n}^{\circ} 3.066 \text { de } 23 \text { de dezembro de } \\
2008\end{array}$ & $\begin{array}{l}\text { Implantação: R\$ 7.000,00 } \\
\text { Custeio/ano: R \$ 24.000,00 }\end{array}$ & $\begin{array}{l}\text { Implantação: R\$ 7.000,00 } \\
\text { Custeio/ano: R \$ 31.200,00 }\end{array}$ \\
\hline Portaria $\mathrm{n}^{\circ} 1.599$ de 09 de julho de 2011 & $\begin{array}{l}\text { Implantação: R\$ 7.000,00 } \\
\text { Custeio/ano: R \$ 25.200,00 }\end{array}$ & $\begin{array}{l}\text { Implantação: R\$ 7.000,00 } \\
\text { Custeio/ano: R } 333.600,00\end{array}$ \\
\hline \multirow[b]{2}{*}{ Portaria $n^{\circ} 978$ de 16 de maio de 2012} & $\begin{array}{l}\text { Implantação: R\$ } 7.000,00 \\
\text { Custeio/ano: R\$ 26.760,00 }\end{array}$ & $\begin{array}{l}\text { Implantação: R\$ } 7.000,00 \\
\text { Custeio/ano: R } \$ 35.760,00\end{array}$ \\
\hline & $\begin{array}{l}50 \% \text { a mais sobre os valores } \\
\text { transferidos referentes às } \\
\text { ESB implantadas } \\
\text { Custeio/ano: } \mathrm{R} \$ 40.140,00\end{array}$ & $\begin{array}{l}50 \% \text { a mais sobre os valores } \\
\text { transferidos referentes às } \\
\text { ESB implantadas } \\
\text { Custeio/ano: } \mathrm{R} \$ 53.640,00\end{array}$ \\
\hline $\begin{array}{l}\text { Portaria } \mathrm{n}^{\circ} 2.539 \text {, de } 26 \text { de setembro de } \\
2019 \\
\text { * Fica vedada a substituição de eSB } \\
\text { nas modalidades I e II composta } \\
\text { por profissionais com carga horária } \\
\text { individual de } 40 \text { horas semanais por eSB } \\
\text { nas Modalidades I-20h e I- } 30 \mathrm{~h}\end{array}$ & $\begin{array}{l}\text { Política Nacional de } \\
\text { Atenção Básica: } \\
\text { Modalidade I-20h: Custeio/ } \\
\text { ano R\$13.380,00. } \\
\text { Modalidade I-30h: Custeio/ } \\
\text { ano: } \$ 20.070,00 .\end{array}$ & Não se aplica \\
\hline
\end{tabular}

Fonte: Elaborado pelos autores. 
A partir de adaptação da metodologia adotada pela Fundação Oswaldo $\mathrm{Cruz}^{10} \mathrm{e}$ do PDR do Instituto de Saúde Coletiva ${ }^{11}$ para a prospecçãoestratégica do sistema de saúde brasileiro, foram projetados três cenários neste estudo:

I. Cenário favorável possível:aumento no repasse de recursos e crescimento da população coberta pelas equipes de saúde bucal na atenção básica estimado em $1 \%$ ao ano com base na série histórica pós-2016. Deste modo, assumiu-se que a cobertura em 2020 será de 42\%, em 2030 de $52 \%$, em 2040 de $62 \%$, alcançando $72 \%$ em 2050. Também haveria atualização dos valores de repasse do governo federal aos municípios para o custeio da saúde bucal na atenção básica acompanhando o IPCA.

Neste estudo, não se adotou o aumento de repasses de recursos baseado nas necessidades de saúde bucal da população brasileira, tendo em vista a vigência da Emenda Constitucional 95/2016, que limita os gastos públicos em 20 anos, condicionando o aumento das despesas federais e repasses à inflação acumulada conforme IPCA. Devido a isso, para projeção dos valores financeiros a serem gastos com as Equipes de Saúde Bucal na Atenção Básica, tomou-se por base a média percentual do IPCA medido pelo IBGE nos últimos 20 anos (1999-2018): 5,94\%.

Este percentual foi aplicado ao atual valor repassado pelo governo federal aos municípios para o custeio mensal das eSB: R\$3.265,25, sendo esta a média dos valores de repasses do Piso da Atenção Básica Variável de Saúde Bucal (ESB modalidade I vinculado a ESF modalidade II: R\$ 2.230,00 por mês; ESB modalidade I vinculado a ESF modalidade I: R\$ 3.345,00 por mês; ESB modalidade II vinculado a ESF modalidade II: R\$2.980,00 por mês. ESB modalidade II vinculado a ESF modalidade I: R \$ 4.470,00 por mês). Assim, em 2020 o valor atualizado seria de R\$ 5.204,80 por equipe. Em 2030, um total de R\$ 7.144,36; em 2040 seria R\$ 9.083,91; alcançando em 2050 o montante de R\$11.023,46.

II. Cenário realista provável: manutenção do atual percentual de cobertura pelas equipes de saúde bucal na atenção básica com base em 2018 (40,2\%) ao longo dos próximos 30 anos. Os valores de repasse do governo acompanhariam para o futuro a tendência da série histórica dos custos informados nas portarias de 2000 a 2019 (32,51\% de crescimento acumulado em quase 20 anos, ou $1,62 \%$ ao ano).

$\mathrm{O}$ atual valor de custeio mensal de R $\$ 3.265,25$ por eSB foi considerado como base para o cálculo da prospecção, aplicando-se sobre este 1,62\%. Em 2020, o valor seria de R $\$ 3.371,03$ por equipe, no ano de 2030 alcançaria $\mathrm{R} \$ 3.899,93$, em 2040 um total de $\mathrm{R} \$ 4.428,83$ e em 2050 o custo de $\mathrm{R} \$ 4.957,73$.

III. Cenário pessimista plausível: diminuição da população coberta pelas equipes de saúde bucal na atenção básica a partir das recentes mudanças na Política Nacional de Atenção Básica (PNAB). Os valores de repasse do governo não sofreriam atualização, mantendo-se os mesmos de 
2018.

O presente estudo dispensou a aprovação de Comitê de Ética em Pesquisa - CEP, tendo em vista que utilizou dados de amplo acesso edomínio público, conforme Resolução № 510 de 7/4/2016 sobre a Ética na Pesquisa na área de Ciências Humanas e Sociais.

\section{RESULTADOS E DISCUSSÃO}

Haverá nos próximos 30 anos, uma profunda mudança na composição demográfica brasileira, com acentuada diminuição da natalidade e aumento da expectativa de vida, caracterizando uma pirâmide populacional similar a encontrada na atualidade em países desenvolvidos. Em 2020, o IBGE estima um total de 211.755.692 habitantes no Brasil, alcançando 224.868.462 em 2030, 231.919.922 em 2040 e 232.933.276 em 2050 ${ }^{12}$ (Gráfico 1)exigindo que o modelo de atenção tenha a atenção básica como lócus privilegiado deste cuidado, dados os seus pressupostos de longitudinalidade e vínculo ${ }^{13}$.

Gráfico 1. Estimativa populacional brasileira para os anos de 2020, 2030, 2040 e 2050.

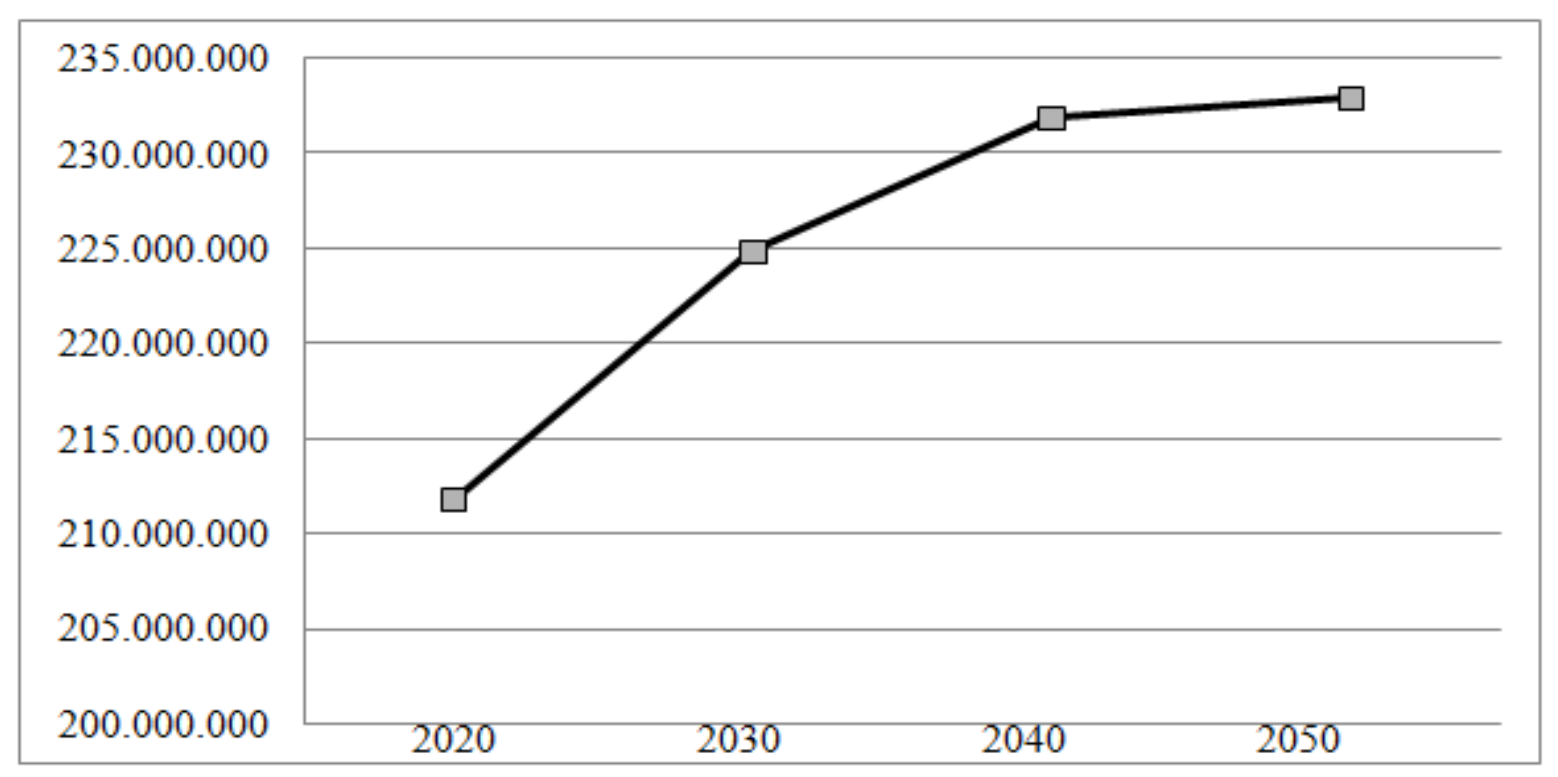

Fonte: IBGE (2019).

\section{Cenário favorável possível}

Neste primeiro cenário, foi previsto o aumento no repasse de recursos, respeitando-se a vigência da Emenda Constitucional 95/2016, que limita os gastos públicos em 20 anos e condicionou o aumento das despesas federais e repasses à inflação acumulada conforme o IPCA. O crescimento da população coberta pelas equipes de saúde bucal na Atenção Básica estimado em 1\% ao ano com base na série histórica pós-2016. A estimativa para a quantidade de eSB seria crescente nos próximos 30 anos com 38.977 equipes em 2030, 47.930 em 2040 e 55.903 em 2050 (Gráfico 2). 
Seguindo a média percentual do IPCA nos últimos 20 anos (1999-2018), para projeção dos valores financeiros a serem gastos com as Equipes de Saúde Bucal na Atenção Básica, considerando apenas o recurso de custeio, obter-se-á um montante de R $\$ 278.465 .720,00$ em 2030, R \$435.391.806,00 em 2040 e R\$607.315.482,00 em 2050 (Gráfico 3).

Gráfico 2. Quantidade de Equipes de Saúde Bucal no Brasil em 2010 e estimativas para 2020, 2030, 2040 e 2050 em cenário favorável possível.

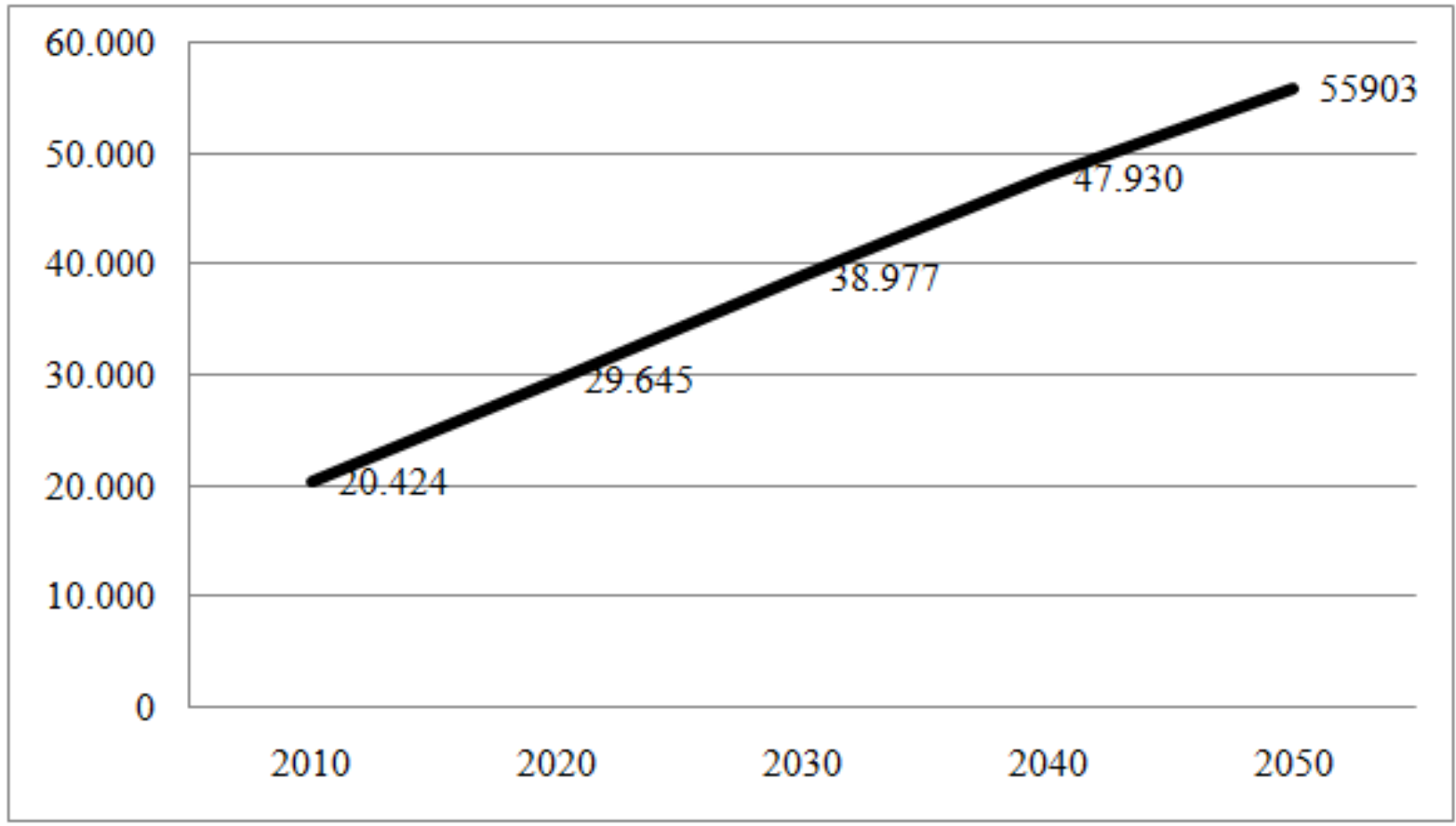

Fonte: Elaborado pelos autores.

Gráfico 3. Estimativa de custos das equipes de saúde bucal no Brasil em 2020, 2030, 2040 e 2050 em cenário favorável possível.

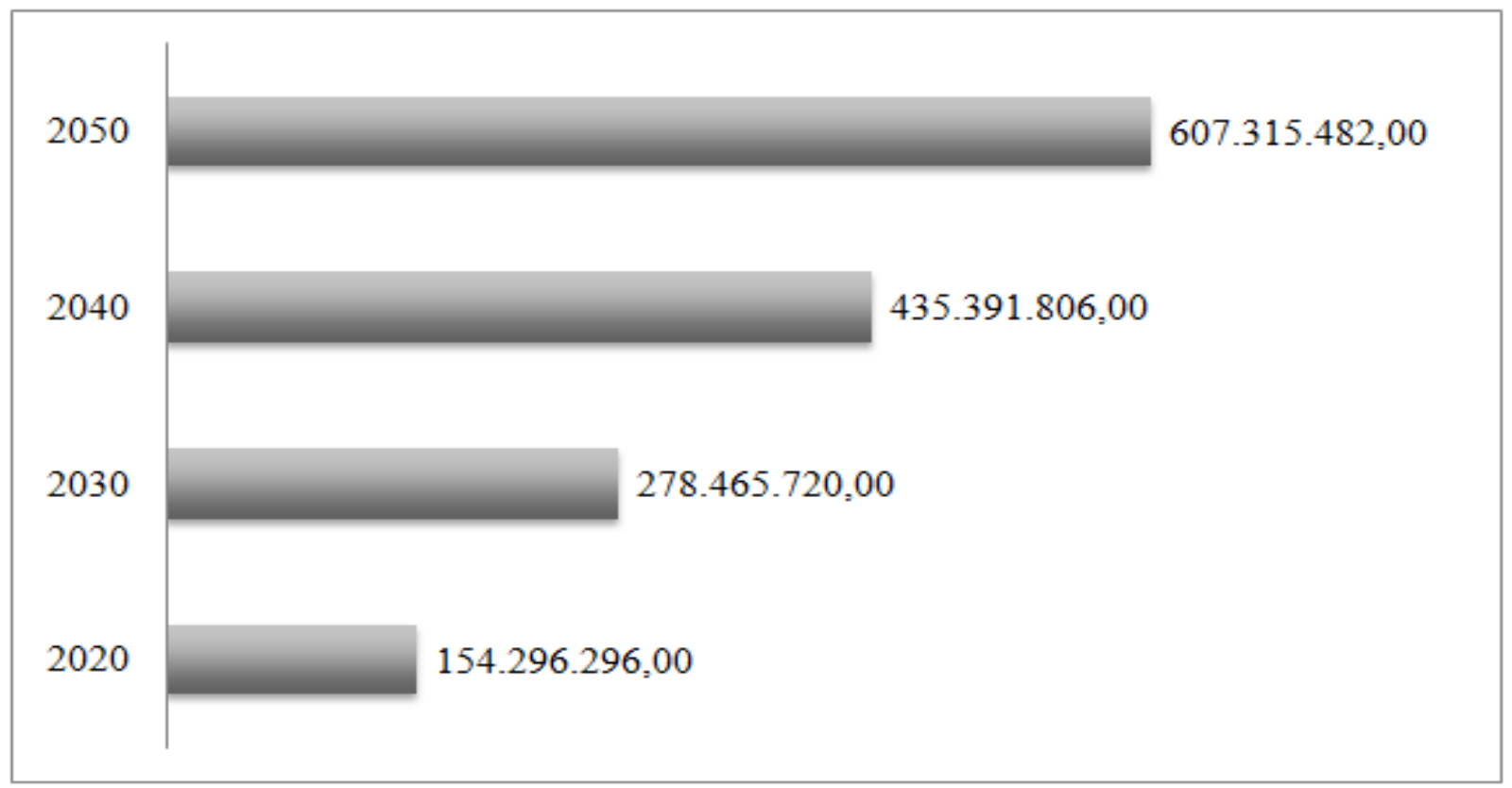

ronte: Elaborado pelos autores. 


\section{Cenário realista provável}

No cenário realista provável, considerou-se a manutenção do atual percentual de cobertura pelas equipes de saúde bucal na atenção básica com base em 2018 (40,2\%) ao longo dos próximos 30 anos. Os valores de repasse do governo acompanhariam para o futuro a tendência da série histórica dos custos informados nas portarias de 2000 a 2019 (32,51\% de crescimento acumulado em quase 20 anos, ou $1,62 \%$ ao ano).

Diferentemente do primeiro cenário, com a manutenção do percentual de cobertura das eSB, considerando o aumento da população brasileira, o número de eSB em 2020 seria de 28.375, em 2030 seria de 30.132, 31.077 em 2040 e 31.213 em 2050 (Gráfico 4).Por conseguinte, os valores estimados para o custeio das equipes será de $\mathrm{R} \$$ 95.652.976,20 em 2020, R\$ 117.512.691,00 em 2030, R\$ 137.634.750,00 em 2040 e R\$ 154.745.626 em 2050 (Gráfico 5).

Gráfico 4. Quantidade de Equipes de Saúde Bucal no Brasil em 2010 e estimativas para 2020, 2030, 2040 e 2050 em cenário realista provável.

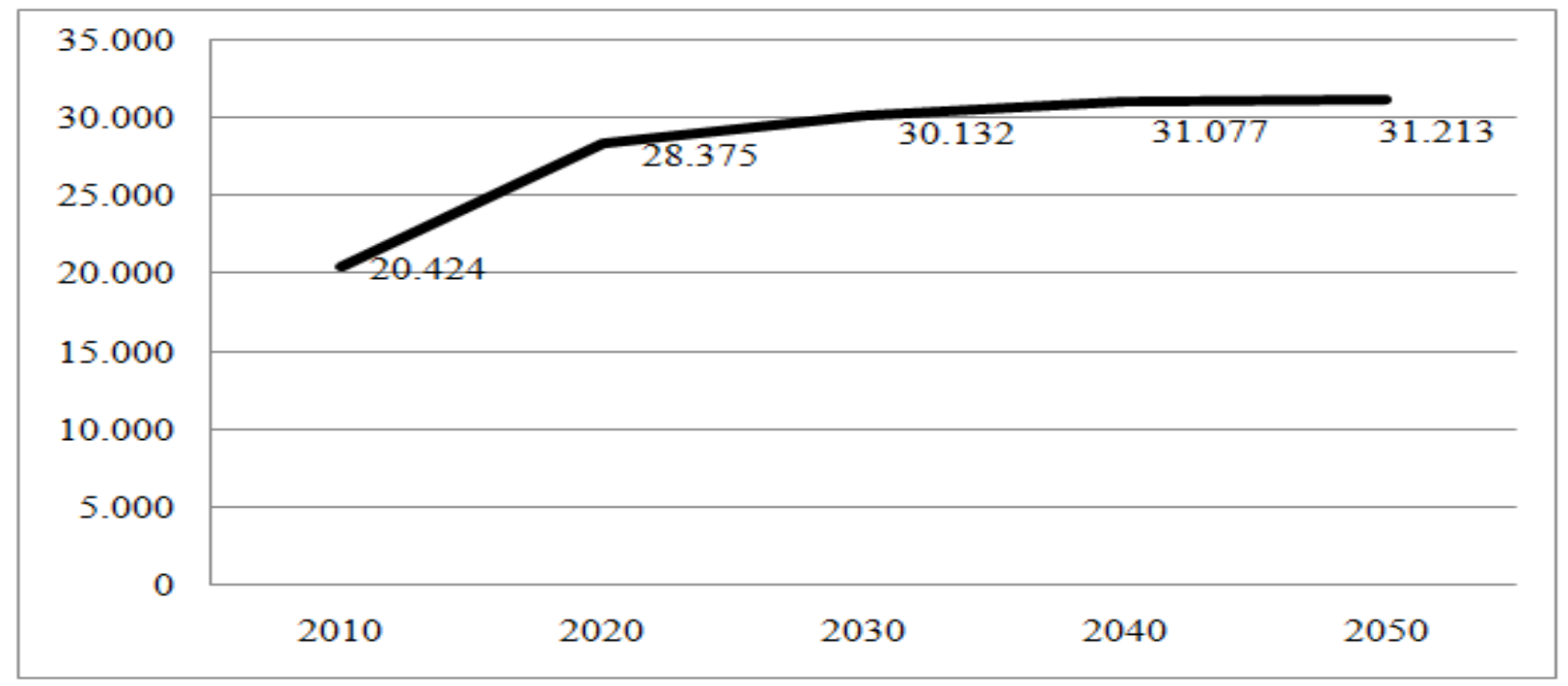

Fonte: Elaborado pelos autores. 
Gráfico 5. Estimativa de custos das equipes de saúde bucal no Brasil em 2020, 2030, 2040 e 2050 em cenário realista provável.

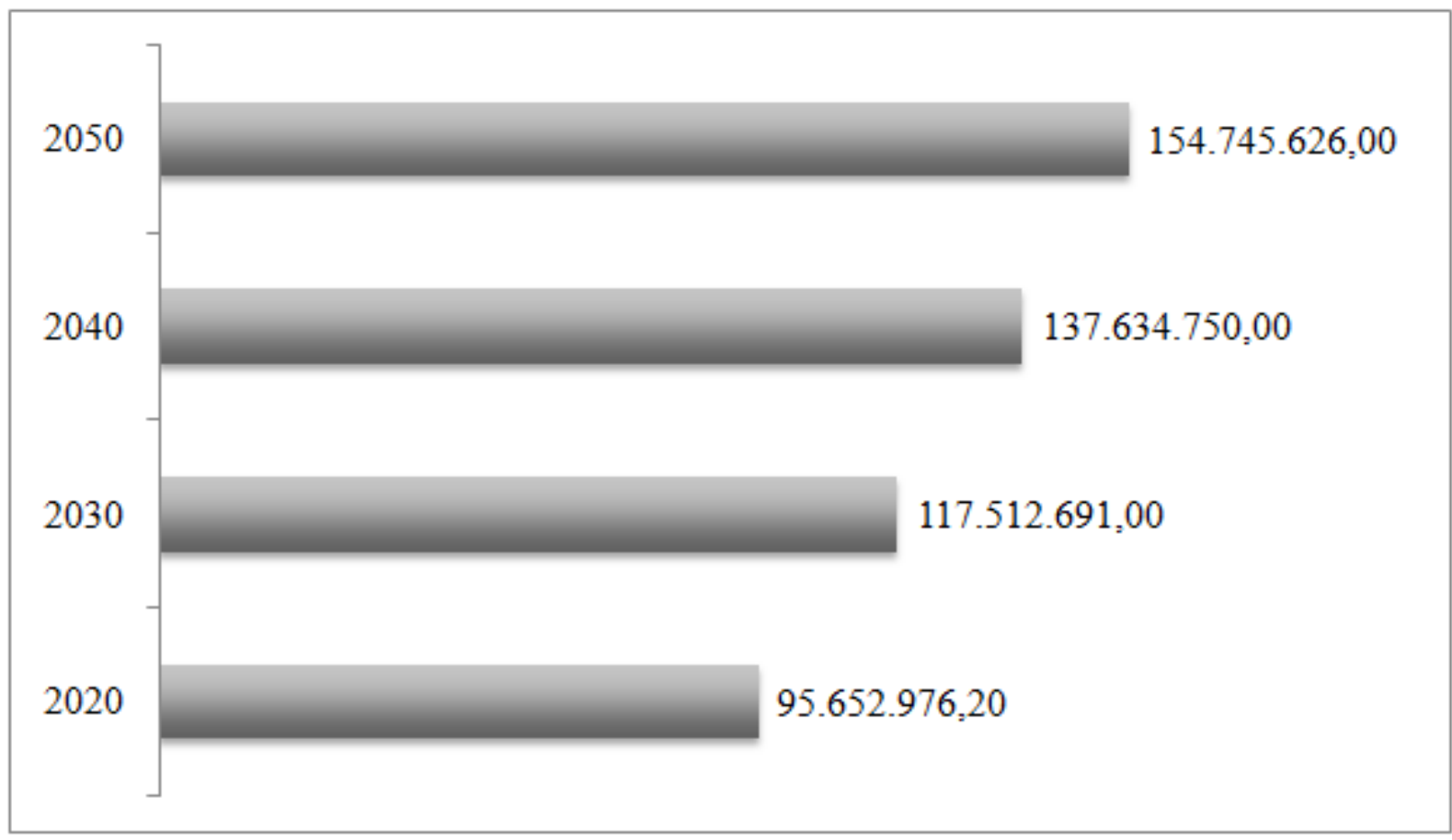

Fonte: Elaborado pelos autores.

\section{Cenário pessimista plausível}

No último cenário vislumbrado, estima-se a diminuição da população coberta pelas equipes de saúde bucal na atenção básica a partir das recentes mudanças na Política Nacional de Atenção Básica (PNAB). Os valores de repasse do governo não sofreriam atualização, a partir da Portaria No 2.539 , de 26 de setembro de 2019 .

Após 27 anos da promulgação das Leis 8.080/1990 e 8.142/1990, pode-se considerar que as políticas desenvolvidas no Sistema Único de Saúde (SUS) foram direcionadas ao fortalecimento da Atenção Primária à Saúde ${ }^{14}$. A Portaria No 2.436, de 21 de setembro de 2017 que aprovou a nova Política Nacional de Atenção Básica no Brasil é apontadacomo definidora de retrocessos como a relativização da cobertura universal, definição de padrões distintos de serviços, recomposição das equipes e do processo de trabalho, segmentação do acesso ao cuidado, desvinculação das equipes dos territórios, desqualificação do trabalho do Agente Comunitário de Saúde (ACS) e Agente de Combate a Endemias (ACE) $)^{14}$.

Distintas entidades vinculadas à luta pela Reforma Sanitária Brasileira como Associação Brasileira de Saúde Coletiva (Abrasco), o Centro Brasileiro de Estudos de Saúde (Cebes) e a Escola Nacional de Saúde Pública (Ensp) manifestaram-se contra a nova PNAB ${ }^{14,15}$. Sobre a Saúde Bucal, Narvai ${ }^{15}$ aponta sobre a perspectiva de término da Brasil Sorridente, como a conhecemos atualmente, como prioridade de governo. 
A Portaria $\mathrm{N}^{\mathrm{o}} 2.539$, de 26 de setembro de 2019, seguindo e referendando o que pressupõe a nova PNAB, diferencia a equipe de atenção primária - eAP da equipe de Saúde da Família eSF. Na eAP, é obrigatório a presença de médicos e enfermeiros, não estando incluído o cirurgião-dentista. Estabelece ainda que podem existir duas modalidades de equipes de Saúde Bucal eSB:

Modalidade I-20h: eSB composta por profissionais com carga horária mínima individual de 20 (vinte) horas semanais e cadastrados em uma mesma Unidade de Saúde, com população adscrita correspondente a 50\% (cinquenta por cento) da população adscrita para uma eSF; ou

Modalidade I-30h: eSB composta por profissionais com carga horária mínima individual de 30 (trinta) horas semanais e cadastrados em uma mesma Unidade de Saúde, com população adscrita correspondente a $75 \%$ (setenta e cinco por cento) da população adscrita para uma eSF.

Não se aplica aos profissionais da eSB na modalidade I com carga horária diferenciada a vedação à participação em mais de uma eSB ou eSF, não sendo hipótese de suspensão de repasse a duplicidade de profissional. ${ }^{16}$

A referida Portaria apresenta uma redução nos valores de incentivo financeiro mensal de custeio para as eSB nestas novas modalidades. Ressalta-se que não há previsão para as equipes de Saúde Bucal na modalidade II, conforme preconizado em Portaria anterior e pela política Brasil Sorridente com a possibilidade de presença dos Técnicos em Saúde Bucal.

Embora já sejam reconhecidos os benefícios de sistemas de saúde organizados a partir da atenção básica, com consenso entre os que formulam as políticas de que este nível de atenção à saúde deve ser fortalecido ${ }^{17}$, um dos cenários que se projeta para a saúde bucal nos próximos anos, aqui denominado de "realista provável", vai na contramão da universalidade como princípio orientador do SUS ${ }^{18}$.

Emerge, neste contexto,a possibilidade de se adotar uma atenção básica seletiva, fundamentada em baixa valorização política, econômica e social, materializada por uma atenção de baixos recursos voltada a pessoas pobres e em regiões pobres ${ }^{19}$.Compromete-se, inclusive, o alcance das metas dos Objetivos de Desenvolvimento Sustentável da Agenda 2030 que preveem a saúde universal ${ }^{20}$.

Por consequência do "cenário pessimista plausível", estima-se que haverá retorno ao modelo de atenção à saúde bucal vivido em décadas anteriores, caracterizado pela exclusão e centrado na assistência curativa individual ${ }^{21}$, o qual acarretará piores condições de saúde bucal no tocante à perda dentária, sobretudo entre adultos e idosos em situação futura no país ${ }^{22}$.

Para além dos benefícios da atenção básica universal diante da situação de saúde bucal da população brasileira, também se estima que quanto maior for a cobertura populacional dos serviços, haverá maior eficiência do gasto público ${ }^{23}$, o que ganha relevância diante das recentes medidas de austeridade fiscal que comprometem os ínfimos recursos financeiros investidos no SUS ${ }^{10}$.

ISSN 1982-8829 Tempus, actas de saúde colet, Brasília, 14(1), 89-102, mar, 2020. Epub Mai/2020 
Assim, o "cenário favorável possível" tem urgência para ser posto em prática, diante do aumento da pobreza e das desigualdades sociais estimadas para o futuro ${ }^{24}$, pois é o único que garantirá ampliação do acesso aos serviços de saúde bucal aos grupos mais vulneráveis e também à classe média que apresenta despesa crescente com saúde em setores privados ${ }^{25}$.

\section{CONSIDERAÇÕES FINAIS}

Vislumbram-se três cenários prospectivos de oferta de serviços de saúde bucal na atenção básica para os próximos trinta anos do SUS. Ao assumir a possibilidade de ampliação da cobertura populacional a um custo suportável mesmo diante das medidas de austeridade fiscal, reforça-se a premissa da vontade política para implementação da política nacional de saúde bucal.

Diante do enfraquecimento do ideal de cidadania ampliada que prevê saúde universal, consequências alarmantes são esperadas para o futuro, as quais incluem dificuldades de acesso à saúde bucal sobretudo entre os grupos sociais mais vulneráveis, com possibilidade de retorno a um modelo de atenção excludente, gerador de iniquidades, agravando a situação de saúde bucal.

\section{REFERÊNCIAS BIBLIOGRÁFICAS}

1. Brasil. Ministério da Saúde. Diretrizes da política de saúde bucal Brasil Sorridente. Brasília; 2004.

2. Brasil. Ministério da Saúde. Sala de apoio à gestão estratégica e participativa. Brasília; 2019.

3. Moysés SJ. Saúde coletiva: políticas, epidemiologia da saúde bucal e rede de atenção odontológica. São Paulo: Artes Médicas; 2013.

4. Moysés SJ. A determinação social da saúde bucal. In: Carrer FCA et al. SUS e saúde bucal no Brasil: gestão e planejamento. São Paulo: Faculdade de Odontologia da USP; 2019.

5. Chaves SCL, Almeida AMFL, Reis CS, Rossi, TRA, Barros SG. Política de saúde bucal no Brasil: as transformações no período 2015-2017. Saúde debate, 43(spe2),76-91; 2018.

6. Rossi TRA, Lorena-Sobrinho JE, Chaves SCL, Martelli PJL. Crise econômica, austeridade e seus efeitos sobre o financiamento e acesso a serviços públicos e privados de saúde bucal. CienSaudeColet, 24(12):4427-4436; 2019.

7. Probst LF, Pucca Júnior GA, Pereira AC, De-Carli AD. Impacto das crises financeiras sobre os indicadores de saúde bucal: Revisão integrativa da literatura.. CienSaudeColet [periódico na internet] (2019/Ago). [Citado em 08/10/2019]. Está disponível em:http://www. cienciaesaudecoletiva.com.br/artigos/impacto-das-crises-financeiras-sobre-os-indicadores-desaude-bucal-revisao-integrativa-da-literatura/17312?id=17312.

Tempus, actas de saúde colet, Brasília, 14(1), 89-102, mar, 2020. Epub Mai/2020 ISSN 1982-8829 
8. Ceccim RB, Kreutz JA. Prospecção de modelos tecnoassistenciais na Atenção Básica: protocolo de pesquia colaborativa multissituada na Educação em Saúde Coletiva. In-formes da atenção básica : aprendizados de intensidade por círculos em rede. 1.ed. Porto Alegre : Rede Unida, 2016. p. 17-30

9. Ipea - Instituto de Pesquisa Econômica Aplicada. Boletim de Análise PolíticoInstitucional. Brasília; 2011.

10. Fundação Oswaldo Cruz. A saúde no Brasil em 2030 - prospecçãoestratégica do sistema de saúde brasileiro: estrutura do financiamento e do gasto setorial [online]. Rio de Janeiro: Fiocruz/Ipea/Ministério da Saúde/Secretaria de Assuntos Estratégicos da Presidência da República, 4;2013. 168 p. ISBN 978-85-8110-018-0.

11. Instituto de Saúde Coletiva da Universidade Federal da Bahia. Plano Diretor 2014-2023. Salvador; 2013.

12. Instituto Brasileiro de Geografia e Estatística. Projeção da população do Brasil e Unidades da Federação por sexo e idade para o período 2010-2060. Rio de Janeiro; 2010.

13. Santos ROM, Romano VF, Engstrom EM. Vínculo longitudinal nasaúde da família: construçãofundamentada no modelo de atenção, práticasinterpessoais e organização dos serviços. Physis, 28(02); 2018.

14. Morosini, MárciaValériaGuimarães Cardoso, Fonseca, Angélica Ferreira e Lima, Luciana Dias de. PolíticaNacional de AtençãoBásica 2017: retrocessos e riscospara o SistemaÚnico de Saúde. Saúdeem Debate [online], 42(116):11-24; 2018 [Acessado 9 Outubro 2019]. Disponívelem: <https://doi.org/10.1590/0103-1104201811601>. ISSN 2358-2898. https://doi. org/10.1590/0103-1104201811601.

15. Narvai PC. O fim do BrasilSorridente? JornalOdonto, 238; 2017.

Disponíveleletronicamenteem: https:/www.jornaldosite.com.br/materias/artigos\&cronicas/ anteriores/paulo\%20capel/artcapel $238 . \mathrm{htm}$

16. Brasil. Portaria $n^{\circ} 2.539$, de 26 de setembro de 2019. Altera as Portarias de Consolidação $\mathrm{n}^{\mathrm{o}}$ 2/GM/MS, de 28 de setembro de 2017, e n ${ }^{\circ} 6$, de 28 de setembro de 2017, parainstituir a equipe de AtençãoPrimária - eAP e disporsobre o financiamento de equipe de SaúdeBucal - eSB com cargahoráriadiferenciada. Brasília; 2019.

17. Giovanella L. Atençãobásicaouatençãoprimária à saúde? Cad. Saúde Pública, 34 (8); 2018 .

18. Mendonça MHM, Matta GC, Gondim R, Giovanella L. Atenção primária à saúde no 
$102 / /$

Brasil: conceitos, práticas e pesquisa. Rio de Janeiro: Editora Fiocruz; 2018.

19. Mendes EV. O cuidado das condiçõescrônicas na atençãoprimária à saúde: o imperativo da consolidação da estratégia da saúde da família. Brasília: Organização Pan-Americana da Saúde; 2012.

20. Organização das Nações Unidas. Documentos temáticos: objetivos de desenvolvimento sustentável 1, 2, 3, 5, 9, 14. Brasília; 2017.

21. Cardoso M, Balducci I, Telles DM, Lourenço EJV, Nogueira Júnior L. Edentulism in Brazil: trends, projectionsandexpectationsuntil 2040. Ciênc. saúde coletiva [Internet]. 21(4): 1239-1246; 2016.

22. Pinto VG. Saúde bucal coletiva. 7. ed. Rio de Janeiro: Guanabara Koogan; 2019.

23. Banco Mundial. Propostas de reformas do sistema único de saúde brasileiro. Washington; 2019.

24. The World Bank. Effectsofthe business cycleon social indicators in LatinAmericaandtheCaribbean: whendreamsmeet reality. Washington; 2019.

25. Instituto Brasileiro de Geografia e Estatística. Pesquisa de orçamentos familiares 20172018: primeiros resultados. Rio de Janeiro; 2019.

Artigo apresentado em outubro de 2019 Artigo aprovado em janeiro de 2020 Artigo publicado em maio de 2020 